\title{
Estudio experimental del rendimiento térmico de un colector solar de tubos evacuados para calentar aire de secado
}

\section{Experimental study of the thermal performance of a solar collector of evacuated tubes for heating drying air}

\author{
FLORES-MONTIJO, Noe A.†* \& CABANILLAS-LOPEZ, Rafael E.
}

Universidad de Sonora, Departamento de Ingeniería Química y Metalurgia, División de Ingeniería, Blvd. Luis Encinas y Rosales SN, Hermosillo, Sonora, C.P. 83000, México

ID 1 ${ }^{\mathrm{er}}$ Autor: Noe A., Flores-Montijo / ORC ID: 0000-0001-9682-5442, CVU CONACYT ID: 920597

ID $1^{\text {er }}$ Coautor: Rafael E., Cabanillas-Lopez / ORC ID: 0000-0003-0739-3348, CVU CONACYT ID: 120410

\section{Resumen}

Se desarrolló un colector solar con tubos evacuados para calentar aire desde temperatura ambiente hasta que tenga la capacidad de usarse en procesos de secado. Se estudió la eficiencia térmica instantánea y global del equipo, así como el comportamiento de la temperatura de salida a diferentes flujos de aire. Se tomaron medidas de las temperaturas del aire de entrada y de salida, la irradiancia y se determinó el flujo másico. Se calculó la cantidad de calor absorbida por el aire y relacionándolo con la irradiancia se obtuvo la eficiencia instantánea para posteriormente determinar la eficiencia global. El dispositivo consiste en un tubo de polipropileno donde se colocaron 5 tubos evacuados. Se hizo fluir aire dentro de cada uno de ellos con un arreglo de un conducto de acero inoxidable con tubería de pared delgada. Para diversos flujos de aire se obtuvieron curvas de comportamiento térmico, donde se observaron incrementos de temperatura del aire entre $30^{\circ} \mathrm{C}$ y $50^{\circ} \mathrm{C}$ alcanzado temperaturas de salida de hasta $90^{\circ} \mathrm{C}$. Se calcularon eficiencias de $40 \%$ a $60 \%$, para temperaturas de operación altas y bajas, respectivamente. El incremento de temperatura resultó con una tendencia lineal, facilitando el diseño de secadores instrumentados y su control.

Secado Solar, Tubos Evacuados, Eficiencia Térmica

\begin{abstract}
A solar collector with evacuated tubes was developed to heat air from room temperature until it has the capacity to be used in drying processes. The instantaneous and global thermal efficiency of the equipment was studied, as well as the behavior of the outlet temperature at different air flows. Measurements of inlet and outlet air temperatures, irradiance were taken, and mass flow were determined. The amount of heat absorbed by the air was calculated and, in relation to the irradiance, instantaneous efficiency was obtained to later determine the overall efficiency. The device consists of a polypropylene tube where 5 evacuated tubes were placed. Air was flowed into each of them with an arrangement of a stainless-steel conduit with thin-walled tubing. For various air flows, thermal behavior curves were obtained, where increases in air temperature between $30^{\circ} \mathrm{C}$ and $50^{\circ} \mathrm{C}$ were observed, reaching outlet temperatures of up to $90^{\circ} \mathrm{C}$. Efficiencies were calculated from $40 \%$ to $60 \%$, for high and low operating temperatures, respectively. The increase in temperature resulted in a linear trend, facilitating the design of instrumented dryers and their control.
\end{abstract}

Solar Drying, Evacuated Tubes, Thermal Efficiency

Citación: FLORES-MONTIJO, Noe A. \& CABANILLAS-LOPEZ, Rafael E. Estudio experimental del rendimiento térmico de un colector solar de tubos evacuados para calentar aire de secado. Revista de Energía Química y Física. 2019. 6-21: 1-8

\footnotetext{
* Correspondencia del Autor (noeflores12@gmail.com)

$\dagger$ Investigador contribuyendo como primer Autor.
} 


\section{Introducción}

El calor es uno de los recursos más importantes para satisfacer nuestras necesidades. Se puede obtener de manera limpia mediante las energías renovables, específicamente, la energía solar. En el proceso de secado se necesita calor para producir aire caliente para facilitar el mecanismo de evaporación de la humedad contenida en una sustancia (Treybal, 1980).

En la industria del secado predominan los alimentos. Dependiendo de cada tipo de alimento se necesita cierta temperatura para secarlo sin dañar sus propiedades. Hay alimentos de baja temperatura; media temperatura (hasta $\left.43^{\circ} \mathrm{C}\right)$ y alta temperatura $\left(82-90^{\circ} \mathrm{C}\right)$ (Maier $\&$ Bakker-Arkema, 2002). El promedio de las temperaturas de secado de algunos alimentos comunes se encontró que fue de $60^{\circ} \mathrm{C}$ (Sharma, Chen, \& Vu Lan, 2009). Otros materiales como la madera necesitan temperaturas desde $80^{\circ} \mathrm{C}$ hasta $115^{\circ} \mathrm{C}$ (Reeb, 1997).

El secado con la energía solar se utiliza desde hace mucho tiempo con la exposición de los alimentos directamente a la luz solar con condiciones ambientales de $30^{\circ} \mathrm{C}$ o más. Para el secado solar se remarca la diferencia de que es necesario utilizar un colector solar para maximizar el aprovechamiento de la energía solar (Sharma et al., 2009).

Los colectores de baja temperatura son utilizados ampliamente para secado de alimentos, debido a que alcanzan temperaturas de hasta $60^{\circ} \mathrm{C}$. Los secadores que utilizan colectores se clasifican en cuatro tipos: directos, indirectos, mixtos e híbridos. (El-Sebaii \& Shalaby, 2012; Fudholi, Sopian, Ruslan, Alghoul, \& Sulaiman, 2010; Sharma et al., 2009). En los secadores solares indirectos (Fig. 1) se calienta el aire en un colector solar externo para después introducirlo en una cámara de secado, evitando exponer la sustancia a la luz solar (Sharma et al., 2009).

Los colectores solares para el secado solar indirecto regularmente son colectores de baja temperatura. Para obtener temperaturas mayores a $60^{\circ} \mathrm{C}$ se quiere analizar el uso de tubos evacuados como medio de calentamiento para el aire de secado.
Los tubos evacuados son colectores de media temperatura, debido a su forma circular pueden calentar aire durante la mayor parte del día, alcanzar temperaturas mayores a $60^{\circ} \mathrm{C}$ y presentar eficiencias entre 50-60\% (Hayek, Assaf, \& Lteif, 2011).

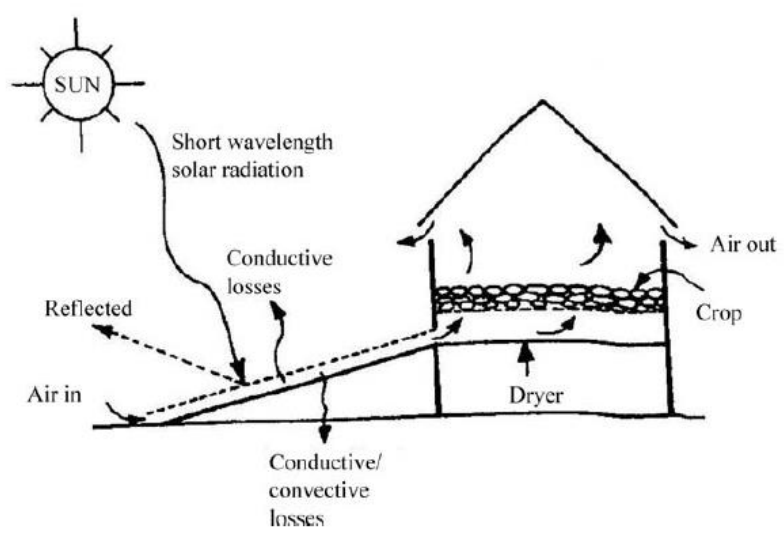

Figura 1 Principio de Trabajo del Secador Solar Indirecto Fuente: (Sharma et al., 2009)

Los colectores solares se ven afectados por las condiciones ambientales en las que se encuentran. Para saber en qué medida, se han hecho estudios para colectores analizando su eficiencia contra un parámetro llamado temperatura reducida, que se calcula con la diferencia de la temperatura de entrada y la temperatura ambiente entre la irradiancia recibida, encontrando funciones lineales con pendiente negativa para la mayoría de los colectores estudiados (Reda, 2017). Cuando se tiene que la temperatura de entrada es la misma que la ambiental surge un problema en el parámetro anterior. Para solucionarlo se cambia la temperatura de entrada por la temperatura promedio del colector y se ajustan los resultados a correlaciones (Gráf. 1). Hayek y colaboradores (2011) presentan un estudio realizado a un colector de tubos evacuados a diferentes condiciones ambientales mostrando que la eficiencia del colector se ajusta a un modelo de segundo grado.

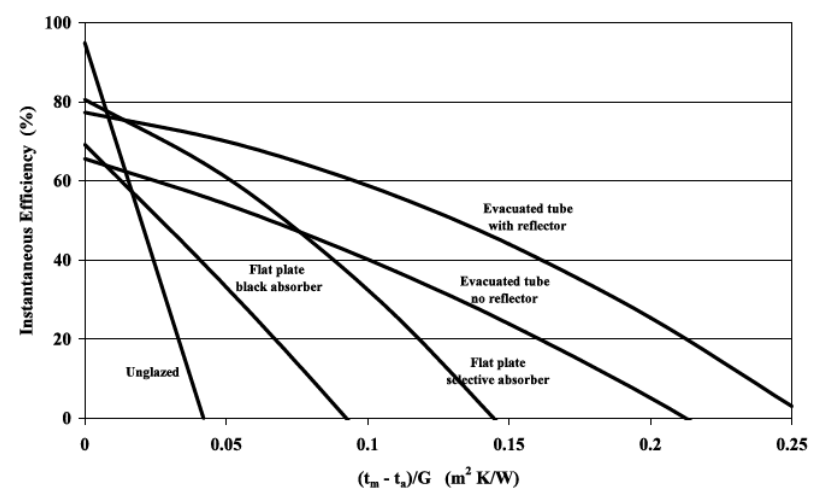

Gráfico 1 Curvas de eficiencia instantánea para varios tipos de colectores solares (MPMSAA, 2009)

FLORES-MONTIJO, Noe A. \& CABANILLAS-LOPEZ, Rafael E. Estudio experimental del rendimiento térmico de un colector solar de tubos evacuados para calentar aire de secado. Revista de Energía Química y Física. 2019. 
Para saber en qué situación se encuentran los colectores solares de tubos evacuados, Sabiha y colaboradores (2015) muestran un panorama sobre el desarrollo de este tipo de colectores, indicando diferentes tecnologías, aplicaciones, rendimiento y otros aspectos relevantes. Kim y Seo (2007) analizaron diferentes configuraciones del interior del tubo absorbedor en un tubo evacuado.

El utilizar un tubo en $U$ en el interior puede traer buenos resultados, sobre todo si se conectan en serie varios tubos para poder alcanzar temperaturas bastante considerables como lo hicieron Wang y su equipo (2014), esto mismo estudiaron Ma y colaboradores (2010), pero analizaron más a fondo como ocurre la transferencia de calor en el tubo evacuado.

Otro aspecto que se ha investigado es el comportamiento térmico de un tubo evacuado al agregar materiales de almacenamiento térmico. Abd-Elhady y colaboradores (2017) estudiaron como afecta el agregar aceite o introducir metales dentro del tubo absorbedor. Otro estudio se hizo agregando materiales de cambio de fase (PCM) en el interior para estudiar su comportamiento térmico (Tyagi, Pandey, Kaushik, \& Tyagi, 2012).

Se encontraron algunos estudios de colectores solares construidos, como el de Yadav y Bajpai (2011) con 40 tubos evacuados. Calientan aire indirectamente a través de un ducto cuadrado, el cual está en contacto con agua caliente que se encuentra en el interior de los tubos evacuados, alcanzando $60^{\circ} \mathrm{C}$ en la temperatura del aire.

Kumar y colaboradores (2013) desarrollaron un colector con 15 tubos evacuados, con la diferencia, que se calienta aire directamente. Hicieron una variación al experimento colocando una superficie reflectiva debajo de los tubos evacuados, encontrando que aumenta el rendimiento del equipo aumentando la temperatura del aire de salida entre 15 y $20^{\circ} \mathrm{C}$. El utilizar un colector solar de tubos evacuados es viable para secado como lo mostraron Lamnatou y colaboradores (2012) en su secador, donde el colector tenía una recirculación de aire para secar diferentes alimentos.
En el presente estudio se analizará un colector solar de tubos evacuados. El objetivo principal es calentar aire y elevar su temperatura tal que pueda llevar a cabo el proceso de secado, tomando como referencia las temperaturas de secado de los alimentos. Además, se analizará su comportamiento térmico, haciendo énfasis en su eficiencia. Se construirá un prototipo del colector solar de tubos evacuados colocándolos en paralelo para calentar aire en condiciones ambientales.

\section{Configuración Experimental}

El colector solar (Fig. 2) está constituido de 5 tubos evacuados situados en un tubo de polipropileno de $0.15 \mathrm{~m}$ de diámetro y de $0.70 \mathrm{~m}$ de largo. Los tubos miden $1.80 \mathrm{~m}$ de largo, el diámetro exterior del tubo de vidrio, $0.0582 \mathrm{~m} \mathrm{y}$ del tubo absorbedor en el interior, $0.0451 \mathrm{~m}$. Tienen una separación de $2.5 \mathrm{~cm}$ entre ellos y una inclinación de 16 grados con respecto a la horizontal. La capa absorbedora de cada tubo mide $1.77 \mathrm{~m}$ dando un área efectiva total de $0.515 \mathrm{~m}^{2}$. Para alimentarse de aire cuenta con un soplador con capacidad de $1 / 35 \mathrm{hp}$ y $12 \mathrm{~V}$ funcionando con una fuente de potencia variable.

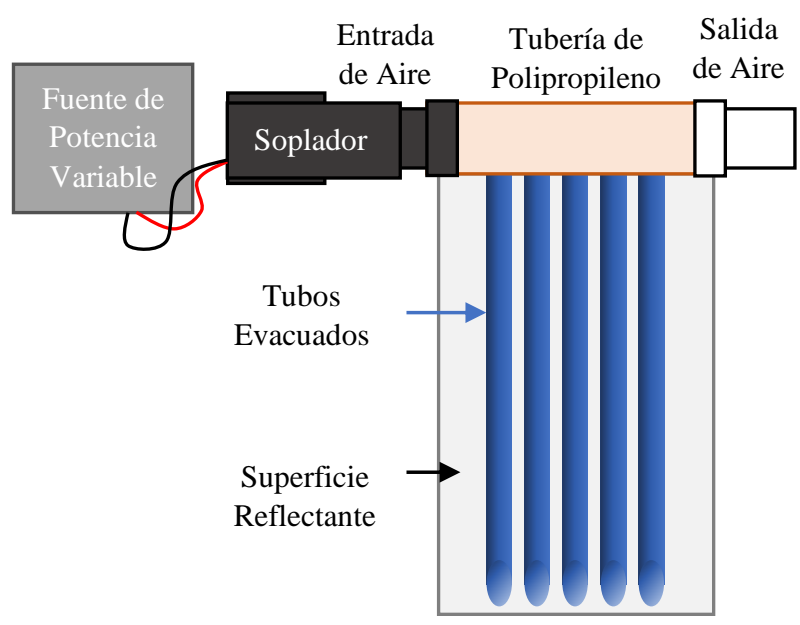

Figura 2 Diagrama de la configuración experimental del colector solar

La tubería se sostiene con dos soportes de madera de $0.50 \mathrm{~m}$. de alto y los tubos evacuados sobre canaletas de acero, que por debajo tienen una superficie reflectante de color blanco. El funcionamiento del colector se muestra en la figura 3. Se alimenta con un sistema de tuberías de acero de pared delgada introducidos $1.40 \mathrm{~m}$ dentro de cada uno de los tubos evacuados, sostenidas por un ducto de acero de $0.075 \mathrm{~m}$ de diámetro y de $0.56 \mathrm{~m}$ de largo que se encuentra en el interior del tubo de polipropileno conectado al soplador.

FLORES-MONTIJO, Noe A. \& CABANILLAS-LOPEZ, Rafael E. Estudio experimental del rendimiento térmico de un colector solar de tubos evacuados para calentar aire de secado. Revista de Energía Química y Física. 2019. 


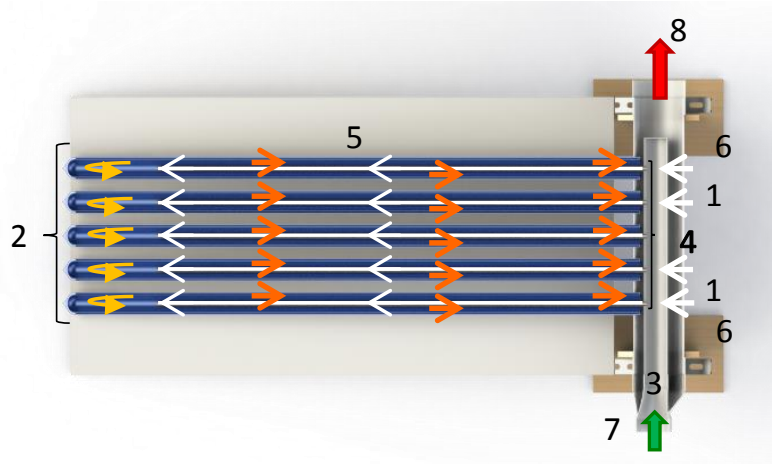

Figura 3 Diseño del Colector: 1) Tubo de Polipropileno; 2) Tubos Evacuados; 3) Ducto de Acero; 4) Tubos de Acero de Pared Delgada; 5) Superficie Reflectante; 6) Soportes; 7) Entrada de Aire; 8) Salida de Aire

La configuración experimental real del colector solar se muestra en la figura 4.

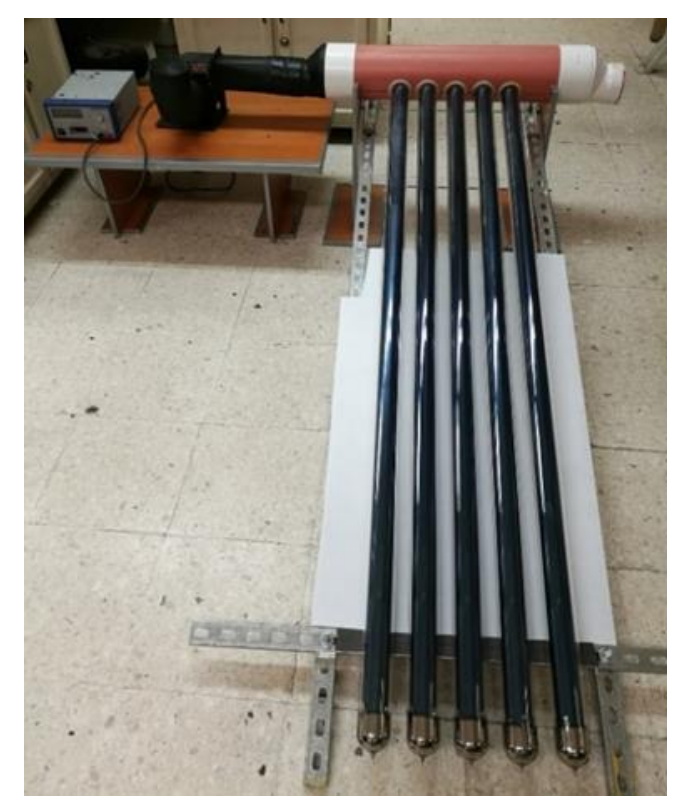

Figura 4 Configuración Experimental del Colector Solar

\section{Metodología}

Los parámetros medidos durante la experimentación fueron la intensidad de la radiación solar y las temperaturas del aire de entrada y de salida, medidas con termopares tipo K. La irradiancia se midió con un piranómetro CMP11 colocado con la misma inclinación que los tubos evacuados (Fig. 5). Las variables fueron registradas con un adquisidor de datos CR10 guardando valores cada minuto durante 11 horas al día.

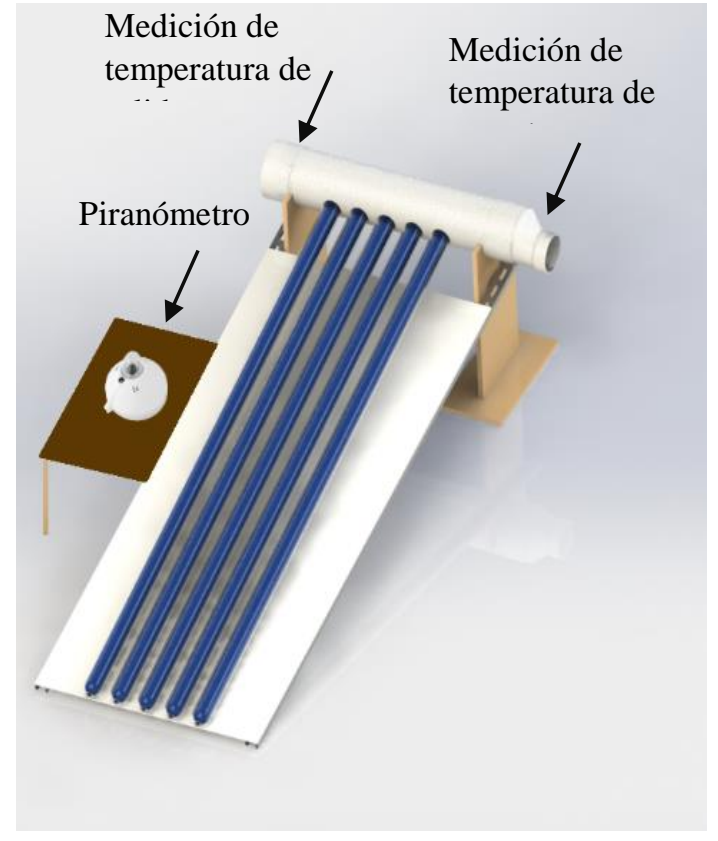

Figura 5 Puntos de medición de las variables en el colector

El flujo de aire se caracterizó midiendo la velocidad del aire con un anemómetro de alambre caliente, Extech 407119A, para diferentes flujos másicos variando el voltaje. Las mediciones se hicieron en diferentes puntos como se muestra en la figura 6 para voltajes de 5 a $11 \mathrm{~V}$, resultando en flujos de 12.7 a $30.4 \mathrm{~kg} / \mathrm{h}$.

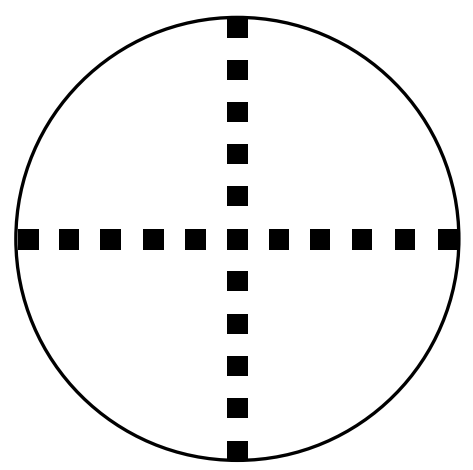

Figura 6 Puntos de medición de la velocidad del aire en la sección transversal de la tubería de salida del colector.

Para encontrar el rendimiento térmico del colector se calculó el calor útil total $(\mathrm{Qu})$, la energía total recibida, la eficiencia instantánea y la global. El calor útil es la energía absorbida en un instante por el aire, calculado con la siguiente expresión:

$\dot{\mathrm{Q}}=\dot{\mathrm{m} C p} \Delta \mathrm{T}=\dot{\mathrm{m} C p}\left(\mathrm{~T}_{\text {out }}-\mathrm{T}_{\text {in }}\right)$

Donde:

$\dot{\mathrm{m}}=$ flujo másico $(\mathrm{kg} / \mathrm{s})$

$\mathrm{Cp}=$ calor específico $(\mathrm{J} / \mathrm{kg} \mathrm{K})$

$\Delta \mathrm{T}=$ Diferencia de temperatura de salida $\mathrm{y}$ entrada $\left({ }^{\circ} \mathrm{C}\right)$

FLORES-MONTIJO, Noe A. \& CABANILLAS-LOPEZ, Rafael E. Estudio experimental del rendimiento térmico de un colector solar de tubos evacuados para calentar aire de secado. Revista de Energía Química y Física. 2019. 
$\dot{\mathrm{Q}}=$ flujo de calor $(\mathrm{J} / \mathrm{s})$ obtiene:

$\mathrm{Al}$ integrar (1) con respecto al tiempo se

$\mathrm{Qu}=\int_{\mathrm{t}_{1}}^{\mathrm{t}_{2}} \mathrm{Q} \mathrm{dt}$

De igual manera, la energía total recibida, se calculó al integrar la radiación instantánea, multiplicada por el área efectiva.

$E=\int_{t_{1}}^{t_{2}} I A d t$

Donde:

$\mathrm{E}=$ Energía total recibida $(\mathrm{J})$

$\mathrm{I}=$ Irradiación solar recibida $\left(\mathrm{J} / \mathrm{m}^{2}\right)$

$\mathrm{A}=$ Área $\left(\mathrm{m}^{2}\right)$

La eficiencia global fue obtenida al relacionar (2) entre (3) y multiplicada por cien.

$\eta=\frac{\mathrm{Qu}}{\mathrm{E}} \times 100$

De acuerdo con la prueba de colectores europea, EN12975, la eficiencia instantánea del colector es dependiente de una correlación, debiendo así utilizar un valor promedio de la temperatura del aire de entrada y de salida (Genersys, 2003).

$\mathrm{T}_{\mathrm{m}}=\frac{\mathrm{T}_{\text {in }}+\mathrm{T}_{\text {out }}}{2}$

Según las características del colector la correlación adecuada para la eficiencia es

$\eta^{\prime}=\eta_{0}-a_{1} \frac{T_{m}-T_{a}}{I}-a_{2}\left(\frac{T_{m}-T_{a}}{I}\right)^{2}$

Donde $\eta_{0}$ representa la eficiencia sin pérdidas de calor; $\mathrm{T}_{\mathrm{a}}$, la temperatura ambiente; $\mathrm{a}_{1} \mathrm{y} \mathrm{a}_{2}$ describen las pérdidas de calor y la temperatura reducida se conoce como $\mathrm{Tr}=$ $\frac{\mathrm{T}_{\mathrm{m}}-\mathrm{T}_{\mathrm{a}}}{\mathrm{I}}$ (Genersys, 2003).

\section{Resultados y Análisis}

El colector solar de 5 tubos evacuados para calentar aire tuvo resultados favorables en elevar la temperatura de entrada en una cantidad considerable. En el gráfico 2 se observa el comportamiento de la temperatura del aire en un día soleado donde se alcanzaron valores cercanos a los $1000 \mathrm{~W} / \mathrm{m}^{2}$. Se utilizó el flujo másico más bajo de $12.7 \mathrm{~kg} / \mathrm{h}$.
La temperatura del aire de entrada estuvo entre los 30 y $40^{\circ} \mathrm{C}$, y fue aumentada hasta casi $60^{\circ} \mathrm{C}$, dando como resultado temperaturas de salida mayores a $90^{\circ} \mathrm{C}$.

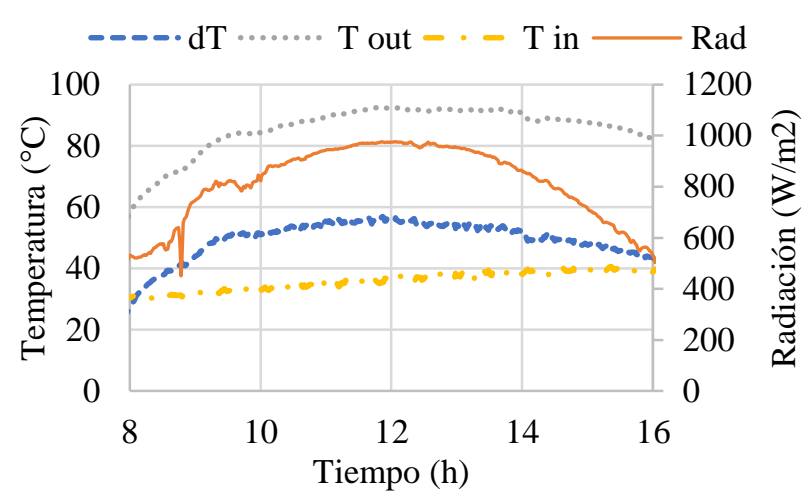

Gráfico 2 Temperaturas de entrada, de salida, diferencia de temperaturas e irradiancia con un flujo de $12.7 \mathrm{~kg} / \mathrm{h}$

Es destacable que la temperatura se mantuvo por arriba de los $60^{\circ} \mathrm{C}$ la mayor parte del día, superando fácilmente la temperatura promedio de secado de los alimentos. Al variar el flujo másico podemos observar que cambia la temperatura de salida del aire (Gráf. 3). Para valores de radiación similares, con máximos superando los $900 \mathrm{~W} / \mathrm{m}^{2}$ y temperaturas de entrada de $35^{\circ} \mathrm{C}$ en promedio se obtuvieron temperaturas de salida entre $60-90^{\circ} \mathrm{C}$ para flujos másicos bajos y $46-77^{\circ} \mathrm{C}$ para flujos másicos altos.

Las temperaturas máximas alcanzadas, entre 13-15 horas, fueron de $91^{\circ} \mathrm{C}, 82^{\circ} \mathrm{C}, 77^{\circ} \mathrm{C}$ y $65^{\circ} \mathrm{C}$ para flujos másicos ascendentes desde el valor menor. De esta manera, se cubre con un amplio rango de temperaturas de materiales para secar con disponibilidad de disponer de una cantidad considerable de aire.

Analizando la variación de la temperaturas máximas de salida con respecto al flujo másico, en el gráfico 4 , se encontró una tendencia lineal en el decrecimiento de la temperatura en un rango de $65-90^{\circ} \mathrm{C}$, pudiendo extenderse algunos grados más. En los limites cercanos de flujo másico cero y en su valor máximo, podría no ser lineal, sin embargo, para los fines de secado esta relación es válida en este rango para determinar un flujo másico dependiendo de la temperatura deseada. 


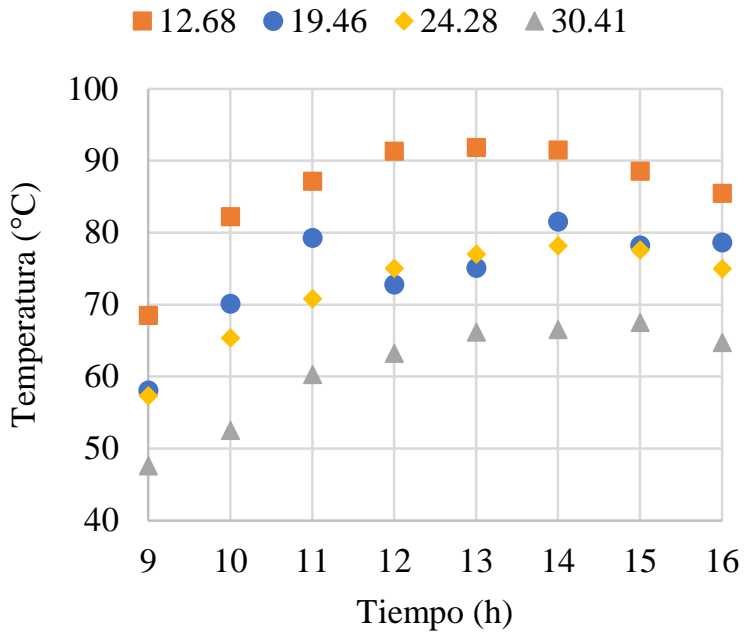

Gráfico 3 Temperatura de salida a diferentes flujos másicos $(\mathrm{kg} / \mathrm{h})$ variando con el paso del tiempo

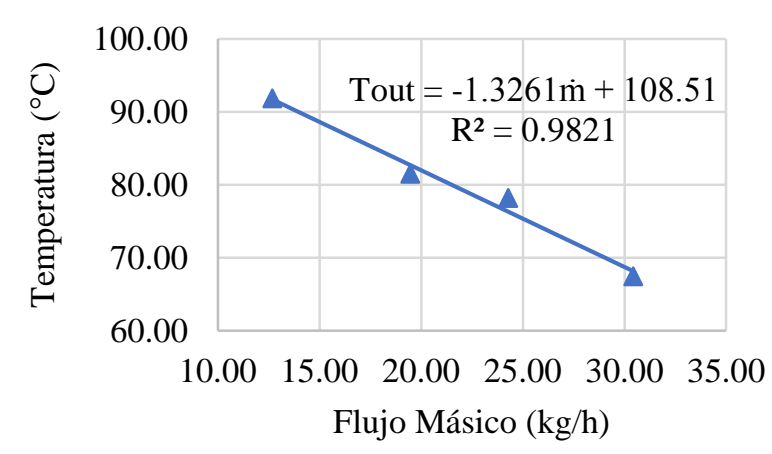

Gráfico 4 Temperatura máxima de salida al variar el flujo másico

El rendimiento del equipo se ve reflejado con la eficiencia de distintas maneras. Al analizar este parámetro en diferentes instantes del día (Gráf. 5) notamos que a diferentes flujos másicos se comparte el mismo comportamiento de una curva cóncava hacia arriba. Los mínimos de estas curvas coinciden con los valores máximos de temperatura, siendo por eso que se presentan mayores pérdidas de calor al medio ambiente. Otro aspecto que destacar es la inercia térmica al final del día, mientras que la radiación va disminuyendo, la eficiencia empieza a aumentar debido al mismo fenómeno.

La eficiencia global (Gráf. 6) al variar con el flujo másico presenta una tendencia polinómica de segundo grado, disminuyendo su pendiente al incrementar el valor del flujo hasta alcanzar su máximo. Para valores de flujo alto las eficiencias son altas, debido a que se tienen menores pérdidas de calor. En flujos bajos, las temperaturas son mayores propiciando pérdidas considerables de calor al ambiente, resultando en eficiencias bajas.

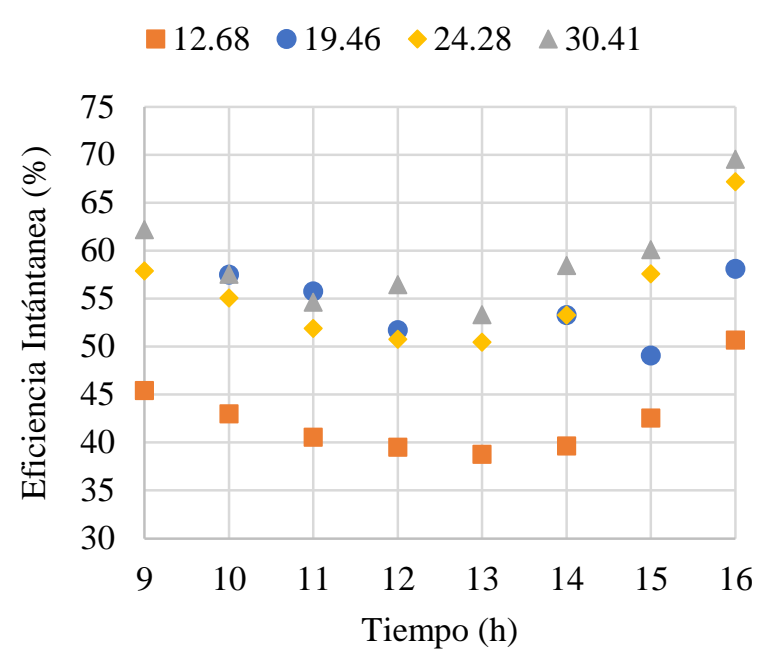

Gráfico 5 Eficiencia instantánea a diferentes flujos másicos $(\mathrm{kg} / \mathrm{h})$ variando con el paso del tiempo

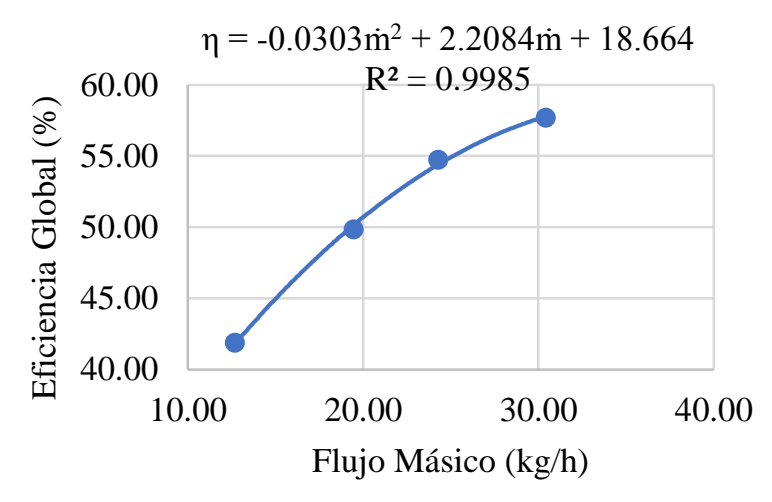

Gráfico 6 Eficiencia global al variar el flujo másico

Según la prueba de colectores europea, EN12975 (Genersys, 2003) al tomar en cuenta las condiciones ambientales y de radiación con respecto a la eficiencia, se debe ajustar a una correlación. En el gráfico 7, la eficiencia para ciertos instantes del día con diferentes flujos másicos y condiciones ambientales tiene una tendencia de un polinomio de segundo orden, como fue indicado por Hayek y colaboradores (2011).

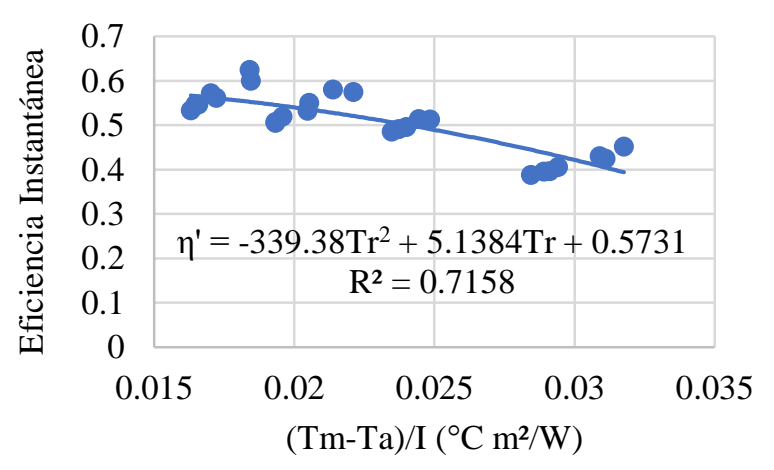

Gráfico 7 Eficiencia instantánea de acuerdo con las variaciones ambientales y de radiación 

instantánea:

Del polinomio obtenido para la eficiencia

$\eta^{\prime}=0.5731+5.1384 \mathrm{~T}_{\mathrm{r}}-339.38 \mathrm{~T}_{\mathrm{r}}^{2}$

Destacando el valor constante, que representa la eficiencia del colector sin pérdidas de calor, siendo de $57.31 \%$.

Analizando la eficiencia global:

$\eta=18.664+2.2084 \dot{m}-0.0303 \dot{m}^{2}$

Si calculamos el punto donde se tiene el máximo, resultando en $36.39 \mathrm{~kg} / \mathrm{h}$, y evaluamos este valor en (8) obtenemos una eficiencia de $58.85 \%$, valor muy similar al obtenido en (7).

\section{Conclusiones}

El colector solar de tubos evacuados con el objetivo de calentar aire para usarse en secado tiene buenos resultados al poder alcanzar temperaturas superiores a los $90^{\circ} \mathrm{C}$. Resalta que supere $\operatorname{los} 60^{\circ} \mathrm{C}$ para diferentes flujos másicos por varias horas al día siendo esto de suma importancia, ya que la temperatura promedio de secado de alimentos es de $60^{\circ} \mathrm{C}$. De esta manera, este secador puede proporcionar una buena cantidad de aire para diferentes materiales a secar con posibilidades de usarse en algunos que requieren altas temperaturas.

La temperatura se puede manipular fácilmente al variar el flujo másico, debido a que tiene un comportamiento lineal como se observa en la siguiente ecuación:

$\mathrm{T}_{\text {out }}=108.51-1.3261 \dot{\mathrm{m}}$

Este aspecto permitirá diseñar un control sencillo para definir una temperatura de salida. Es destacable que este colector construido con 5 tubos evacuados haya obtenido resultados similares a otros estudios reportados con mayor número de tubos. La eficiencia del colector fue la esperada, situándose en el rango reportado para tubos evacuados de 50-60\%.

Se recomienda hacer algunas modificaciones para trabajo futuro. $\mathrm{Si}$ se construye uno colector con un mayor número de tubos evacuados se podrían obtener cantidades considerables de aire a temperaturas altas para diferentes procesos, incluyendo el secado.
También al aislar de manera efectiva el colector y operando preferentemente a flujos másicos altos su eficiencia podría mejorarse.

\section{Agradecimiento}

A la Universidad de Sonora y al Centro Mexicano de Innovación en Energía Solar (CeMIE Sol) por la financiación de este proyecto.

\section{Referencias}

Abd-Elhady, M. S., Nasreldin, M., \& Elsheikh, M. N. (2017). Improving the performance of evacuated tube heat pipe collectors using oil and foamed metals. Ain Shams Engineering Journal. doi:10.1016/j.asej.2017.10.001

El-Sebaii, A. A., \& Shalaby, S. M. (2012). Solar drying of agricultural products: A review. Renewable and Sustainable Energy Reviews, 16(1), 37-43. doi:10.1016/j.rser.2011.07.134

Fudholi, A., Sopian, K., Ruslan, M. H., Alghoul, M. A., \& Sulaiman, M. Y. (2010). Review of solar dryers for agricultural and marine products. Renewable and Sustainable Energy Reviews, 14(1), 1-30. doi:10.1016/j.rser.2009.07.032

Genersys, P. (2003). Collector test according to EN 12975-1,2. Freiburg.

Hayek, M., Assaf, J., \& Lteif, W. (2011). Experimental investigation of the performance of evacuated-tube solar collectors under eastern mediterranean climatic conditions. Energy Procedia, 6, 618-626. doi:10.1016/j.egypro.2011.05.071

Kim, Y., \& Seo, T. (2007). Thermal performances comparisons of the glass evacuated tube solar collectors with shapes of absorber tube. Renewable Energy, 32(5), 772795. doi:10.1016/j.renene.2006.03.016

Kumar, A., Kumar, S., Nagar, U., \& Yadav, A. (2013). Experimental Study of Thermal Performance of One-Ended Evacuated Tubes for Producing Hot Air. Journal of Solar Energy, 2013, 1-6. doi:10.1155/2013/524715 
Lamnatou, C., Papanicolaou, E., Belessiotis, V., \& Kyriakis, N. (2012). Experimental investigation and thermodynamic performance analysis of a solar dryer using an evacuated-tube air collector. Applied Energy, 94, 232-243. doi:10.1016/j.apenergy.2012.01.025

Ma, L., Lu, Z., Zhang, J., \& Liang, R. (2010). Thermal performance analysis of the glass evacuated tube solar collector with U-tube. Building and Environment, 45(9), 1959-1967. doi:10.1016/j.buildenv.2010.01.015

Maier, D. E., \& Bakker-Arkema, F. W. (2002). Grain Drying Systems. Retrieved 11 June 2019, from

https://fyi.uwex.edu/energy/files/2016/09/Grain -drying-Systems-GEAPS-2002-secured.pdf

MPMSAA, S. (2009). Large Scale Solar Thermal Systems Design Handbook.

Reda, F. (2017). Solar Assisted Ground Source Heat Pump Solutions, 9-16. doi:10.1007/978-3319-49698-6

Reeb, J. E. (1997). Drying Wood (No. 55). Kentucky.

Sabiha, M. A., Saidur, R., Mekhilef, S., \& Mahian, O. (2015). Progress and latest developments of evacuated tube solar collectors. Renewable and Sustainable Energy Reviews. doi:10.1016/j.rser.2015.07.016

Sharma, A., Chen, C. R., \& Vu Lan, N. (2009). Solar-energy drying systems: A review. Renewable and Sustainable Energy Reviews, 13(6-7),

1185-1210. doi:10.1016/j.rser.2008.08.015

Treybal, R. (1980). Operaciones de Transferencia de Masa (2nd ed.). McGraw Hill.

Tyagi, V. V., Pandey, A. K., Kaushik, S. C., \& Tyagi, S. K. (2012). Thermal performance evaluation of a solar air heater with and without thermal energy storage. Journal of Thermal Analysis and Calorimetry, 107(3), 1345-1352. doi:10.1007/s10973-011-1617-3
Wang, P. Y., Guan, H. Y., Liu, Z. H., Wang, G. S., Zhao, F., \& Xiao, H. S. (2014). High temperature collecting performance of a new allglass evacuated tubular solar air heater with Ushaped tube heat exchanger. Energy Conversion and Management, 77, 315-323. doi:10.1016/j.enconman.2013.08.019

Yadav, A., \& Bajpai, V. K. (2011). An Experimental Study on Evacuated Tube Solar Collector for Heating of Air in India. International Journal of Mechanical, Aerospace, Industrial, Mechatronic, and Manufacturing Engineering, 5(7), 1188-1193. doi:10.5281/zenodo.1083537 